\section{A viabilidade da pratica de treinamento físico em pacientes com leucemia aguda: uma revisão sistemática}

\section{The feasibility of the practice of physical exercise in patients with acute leukemia: a systematic review}

\author{
Alan Moraes ${ }^{1,2,3}$ \\ Claudio Battaglini 4,5 \\ Douglas Contini Smielewski ${ }^{1,3}$ \\ Ariany Marques Vieira ${ }^{1,3}$ \\ Magnus Benetti ${ }^{1,3,4,6}$
}

\section{RESUMO}

O tratamento da leucemia acarreta efeitos adversos aos pacientes e sequelas que se manifestam de forma aguda (durante o tratamento) e também em longo prazo (pós-tratamento). Pesquisas com pacientes oncohematológicos vêm mostrando que o treinamento físico possa ser capaz de minimizar os efeitos adversos do tratamento quimioterápico do câncer. Este estudo objetiva verificar a viabilidade de um programa estruturado de treinamento físico especificamente em pacientes com leucemia mielóide aguda e leucemia linfoide aguda durante o tratamento inicial da doença. Foi efetuada uma revisão de literatura nas bases de dados computadorizadas LILACS, EMBASE, MEDLINE, SPORTDiscus, CINAHL, Cochrane e PEDro. Como principais critérios de inclusão estudos com mais de $50 \%$ dos pacientes com diagnóstico de leucemia aguda, artigos originais em que o treinamento físico fosse a principal intervenção utilizada e com avaliações objetivas dos desfechos. Foram selecionados seis artigos, os quais analisavam a viabilidade e eficácia do treinamento físico. Treinamento físico demonstrou melhorar ou manter os níveis de fadiga, além de relatada melhorias na angustia, ansiedade, qualidade de vida e depressão, assim como no desempenho físico nessa população. Em conclusão, os estudos apontam para a viabilidade e segurança do treinamento físico executado por pacientes oncohematológicos, todavia, mais pesquisas são necessárias.

\section{PALAVRAS-CHAVE}

Treinamento físico; Leucemia; Revisão.

\begin{abstract}
The treatment of leukemia causes adverse side effects to patients and sequels manifested as an acute (during treatment) and in the long time (post-treatment). Researches with bematologic patients have shown that physical exercise may be able to minimize the possible side effects of cancer treatment. This study aimed to investigate the viability of an exercise program specifically for patients with acute myeloid leukemia (AML) and acute lymphocytic leukemia (ALL) during the initial treatment of the disease. We conducted a literature review in computerized databases LILACS, EMBASE, MEDLINE, SPORTDiscus, CINAHL, Cochrane and PEDro. The main criterion for inclusion was the studies have more than $50 \%$ of patients diagnosed with acute leukemia, original articles on which the exercise was the main intervention employed and objective evaluations of outcomes. Six articles were selected, which analyzed the effectiveness of physical exercise on quality of life, fatigue, nausea, depression, anxiety, respiratory capacity, endurance and physical performance, these factors present in most individuals with acute leukemia, and promising improvements were noted in several of them. In conclusion, the results of these preliminary studies are encouraging and present promising results regarding the potential benefits that exercise may provide patients with hematological cancers.
\end{abstract}

Rev Bras Ativ Fis Saúde p. 277-285 DOI

http://dx.doi.org/10.12820/rbafs.v.19n3p277

1Universidade do Estado de Santa Catarina (UDESC), Centro de Ciências da Saúde e do Esporte (CEFID). Florianópolis, SC-Brasil.

2 Universidade do Sul de Santa Catarina (UNISUL), Curso de Medicina. Palhoça, SCBrasil.

3Núcleo de Cardiologia e Medicina do Exercício, Universidade do Estado de Santa Catarina. Florianópolis, SC-Brasil.

4 University of North Carolina at Chapel Hill (UNC), Departamento de Ciências do Desporto e Exercício (Especialização Fisiologia Exercício). Chapel Hill, NC - EUA.

5 UNC Lineberger Comprehensive Cancer Center. Prevenção e Controle de Câncer (UNC). Chapel Hill, NC - EUA.

6 Clínica de Prevenção e Reabilitação Cardiosport. Florianópolis, SC - Brasil.

\title{
KEYWORDS
}

Physical exercise; Leukemia; Revision. 


\section{INTRODUÇÃO}

A Leucemia é caracterizada pela diferenciação limitada de mieloblastos ou linfoblastos, proliferação desregulada de células anormais e produção diminuída de células sanguíneas normais ${ }^{1,2}$. Os mecanismos para gênese da mesma ainda são pouco compreendidos ${ }^{1,2}$ e pacientes no mesmo estágio da doença normalmente apresentam respostas diferentes ou prognósticos variados ${ }^{3}$.

Só no Brasil, para 2013, estimam-se 4.570 casos novos de leucemia em homens e 3.940 em mulheres. Esses valores correspondem a um risco de cinco novos casos a cada 100 mil homens e quatro a cada 100 mil mulheres ${ }^{4}$.

O tratamento da leucemia, assim como para qualquer outro tipo de câncer é bastante complexo. Seu procedimento padrão envolve a utilização de quimioterapia seguida de, na maioria dos casos, transplante de medula óssea ${ }^{5}$. Apesar de 20-70\% de todos os pacientes obterem a cura clínica, dependendo da apresentação da doença, as sequelas em longo prazo da imunossupressão, toxicidade da quimioterapia e presença da doença enxerto-versus-hospedeiro (DEVH) debilitam um grande número de pacientes ${ }^{6}$. Os principais efeitos adversos observados são fadiga debilitante ${ }^{7,8}$, náuseas, vômitos, diarreia ${ }^{9}$, anemia, descondicionamento físico ${ }^{10}$, depressão, ansiedade e perda de qualidade de vida $^{10,11}$. Autores mostram que problemas como fadiga, ansiedade e depressão persistem além do período de recuperação aguda após o tratamento ${ }^{12,13}$.

Contudo, pesquisas com pacientes oncohematológicos vêm mostrando que o treinamento físico aparentemente é capaz de mitigar alguns dos possíveis efeitos adversos do tratamento do câncer, tais como a fadiga, perda de massa corporal, diminuição da capacidade física e cardiovascular, assim como aumentar a qualidade de vida destes indivíduos ${ }^{14-18}$ durante o tratamento da doença. Para evitar o desconforto, os pacientes podem diminuir o nível de atividade física. Todavia, o repouso prolongado pode maximizar o catabolismo muscular, diminuição do tônus e desempenho físico, consequentemente, impactando na qualidade de vida ${ }^{19,20}$. Estudos recentes têm afirmado que mais pesquisas são necessárias para determinar a viabilidade de um programa de treinamento físico e como ele pode ser aprimorado para pacientes com câncer hematológico ${ }^{21,22}$.

Postula-se que uma intervenção de treinamento físico pode atenuar os sintomas de tratamento, aumentar a função fisiológica e psicológica e melhorar a qualidade de vida global em doentes adultos e idosos com leucemia aguda em tratamento ${ }^{23}$. Sendo assim, baseada nos efeitos positivos de um programa estruturado de treinamento físico e na necessidade de orientação sobre seu benefício, essa revisão sistemática da literatura visa verificar a viabilidade deste, especificamente em pacientes com leucemia mielóide aguda (LMA) e leucemia linfoide aguda (LLA).

\section{MÉTODOS}

A metodologia aplicada foi baseada nas recomendações da Colaboração Cochrane $^{24}$, a busca dos artigos originais foi determinada com base no acrônimo PICO (Paciente, Intervenção, Controle e Desfecho-Outcome), as palavraschave utilizadas foram: "exercise" or "physical activity" or "endurance" and "hematological cancer" or "leukemia", assim como "exercício físico" ou "ati- 
vidade física" ou "exercício físico aeróbio" ou "treinamento físico" ou "treinamento de força" e "câncer hematológico" ou "leucemia".

As bases de dados utilizadas foram: LILACS, EMBASE, MEDLINE, SPORTDiscus, CINAHL, Cochrane e PEDro. Também foram contatados os grupos de pesquisa em treinamento físico e câncer para saber se existiam estudos efetuados e não publicados que pudessem ser inseridos nesta revisão. Como critérios de inclusão foram considerados: estudos com mais de 50\% dos pacientes com diagnóstico de leucemia aguda, com idade igual ou superior a 18 anos, sendo artigos originais em que o treinamento físico fosse a principal intervenção utilizada e que houvesse avaliações objetivas dos desfechos. Pela escassez de estudos foram consideradas não apenas os ensaios clínicos, mas também estudos de grupo único, desde que atendessem aos critérios de inclusão.

\section{Seleção dos estudos}

Os avaliadores tiveram acesso individualmente a todos os artigos encontrados, em uma reunião de consenso estabeleceram quais estudos entraram na revisão.

\section{Extração de dados}

Foi realizada uma ampla revisão qualitativa dos estudos de intervenção, de onde foram extraídas as informações sobre a concepção do estudo, dados demográficos, tipo de câncer, estrutura e tipo de intervenção (treinamento físico aeróbio, de força ou concorrente - exercício físico aeróbio e de força concomitantes), grau de adesão, duração da intervenção e principais resultados.

\section{RESULTADOS}

Foram encontradas 949 potenciais citações, sendo selecionados seis estudos que contemplaram os critérios de inclusão e utilizados para realização deste trabalho. Após primeira leitura foram incluídos 118 artigos relacionados ao tema, entretanto, 112 estudos foram excluídos por que contavam com pacientes em sua maioria com tumores sólidos, não continham treinamento físico como sendo a principal intervenção ou não utilizaram medidas objetivas da intervenção física, conforme a Figura 1.

A Tabela 1 sintetiza as características dos artigos originais que compõe a amostra, bem como procedimentos metodológicos e principais resultados. A amostra dos estudos variou de 10 a 52 indivíduos, de ambos os sexos, com período de treinamento tendo duração de 17 dias a sete semanas, sendo realizadas entre três e quatro sessões semanais. Quanto às intervenções estudadas pelos autores, houve um predomínio de treinamento concorrente (treinamento aeróbio combinado com treinamento de força), com exceção de Cunningham et al. ${ }^{25}$ e Chang et al. ${ }^{26}$ que verificaram exclusivamente o efeito do treinamento de força e treinamento aeróbio, respectivamente. A intensidade dos treinamentos aeróbios foi monitorada pela frequência cardíaca dos pacientes, porém não foram aplicados métodos precisos na mensuração (teste ergoespirométrico). Dois estudos ${ }^{27,28}$ acrescentaram o treinamento de flexibilidade na intervenção. 
FIGURA 1 - Fluxograma dos artigos encontrados.

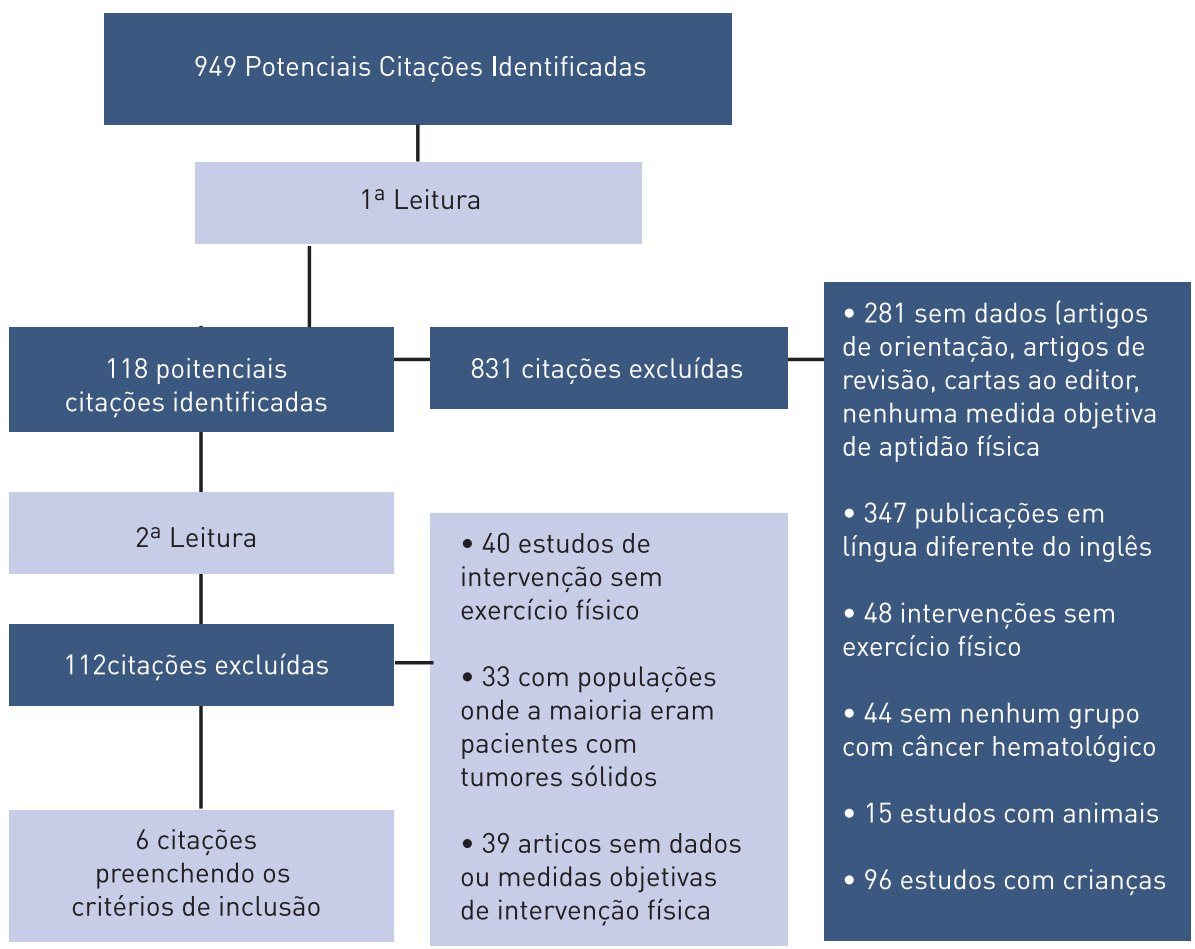

TABELA 1 - Descrição das características e resultados dos estudos envolvendo LA

\begin{tabular}{|c|c|c|c|c|c|c|c|}
\hline $\begin{array}{l}\text { Citação/ Revista/ } \\
\text { Fator de Impacto }\end{array}$ & $\begin{array}{l}\text { Tipo de } \\
\text { estudo }\end{array}$ & Amostra & Idade & $\begin{array}{l}\text { Intervenção/ } \\
\text { Treinamento físico }\end{array}$ & Duração & $\begin{array}{l}\text { Frequência/ } \\
\text { Intensidade }\end{array}$ & Resultados/Atrito \\
\hline $\begin{array}{l}\text { Cunningham } \\
\text { et al. }(1986)^{25} \\
\text { / Journal of } \\
\text { Parenteral and } \\
\text { Enteral Nutrition } \\
/ 3,285\end{array}$ & $\begin{array}{l}\text { Ensaio } \\
\text { clínico } \\
\text { randomizado }\end{array}$ & $\begin{array}{l}40 \text { indivíduos } \\
\text { com LMA e } \\
\text { LLA pós TCTH }\end{array}$ & $\begin{array}{l}\text { GC } 22,5 \\
\text { anos (15- } \\
41) ; \\
\text { GPT3 20,8 } \\
\text { anos (14- } \\
33) ; \\
\text { GPT5 26 } \\
\text { anos (15- } \\
38 \text { ) }\end{array}$ & $\begin{array}{l}3 \text { grupos. } \\
\text { GC sem terapia; } \\
\text { GPT3 com } \\
\text { fisioterapia } 3 x / \text { sem; } \\
\text { GPT5 com } \\
\text { fisioterapia } 5 x / \text { sem }\end{array}$ & $\begin{array}{l}5 \\
\text { semanas }\end{array}$ & $\begin{array}{l}\text { Grupos PT3 e PT5: } \\
30 \text { min de TF com } \\
15 \text { repetições em } 09 \\
\text { grupos musculares } \\
\text { diferentes. } \\
\text { Intensidade não } \\
\text { informada }\end{array}$ & $\begin{array}{l}\text { - proteína muscular no GC. } \\
10 \text { indivíduos não } \\
\text { concluíram o estudo (05 por } \\
\text { motivo não relatado; } 05 \text { por } \\
\text { complicações médicas) } \\
\text { Autor não relatou desistências } \\
\text { relacionadas ao EX }\end{array}$ \\
\hline $\begin{array}{l}\text { Chang et } \\
\text { al. }(2008)^{26} / \\
\text { Journal of Pain } \\
\text { and Symptom } \\
\text { Management / } \\
\text { 2,423 }\end{array}$ & $\begin{array}{l}\text { Ensaio } \\
\text { clínico } \\
\text { randomizado }\end{array}$ & $\begin{array}{l}24 \text { indivíduos } \\
\text { com LMA - em } \\
\text { tratamento }\end{array}$ & $\begin{array}{l}\text { GI: } \\
49,4 \pm 15,3 \\
\text { anos; } \\
\text { GC: } \\
53,3 \pm 13,6 \\
\text { anos }\end{array}$ & $\begin{array}{l}2 \text { grupos. } \\
\text { GC: visita da } \\
\text { enfermagem; } \\
\text { GI: caminhada } \\
\text { supervisionada }\end{array}$ & $\begin{array}{l}3 \\
\text { semanas }\end{array}$ & $\begin{array}{l}\text { GI: } 5 x / \text { sem, } 12 \mathrm{~min} \\
\text { Intensidade: FC } \\
\text { Repouso + } 30 \text { BPM }\end{array}$ & $\begin{array}{l}\text { GI: distância total } \\
\text { percorrida (capacidade } \\
\text { funcional geral), - }{ }^{-} \text {angústia, } \\
\leftrightarrow \text { níveis de fadiga em todo } \\
\text { o estudo } \\
\text { GC: - na distância total } \\
\text { percorrida (capacidade } \\
\text { funcional geral) e em fadiga. } \\
02 \text { complicações da doença. } \\
\text { Nenhum evento indesejável } \\
\text { devido ao EX relatado }\end{array}$ \\
\hline $\begin{array}{l}\text { Battaglini et } \\
\text { al. }(2009)^{23} / \\
\text { Integrative } \\
\text { Cancer Therapies } \\
\text { / 2,136 }\end{array}$ & Grupo único & $\begin{array}{l}10 \text { indivíduos } \\
\text { com } \\
\text { LMA - em } \\
\text { tratamento }\end{array}$ & $\begin{array}{l}35,7 \pm 8,9 \\
\text { anos }\end{array}$ & $\begin{array}{l}\text { Grupo único, EX } \\
\text { supervisionado: } \\
\text { alongamento + TC } \\
\text { (cicloergômetro e/ } \\
\text { ou esteira + TF) + EX } \\
\text { não supervisionado } \\
\text { aeróbio domiciliar } \\
\text { (caminhada) } \\
\text { durante as } 2 \text { últimas } \\
\text { semanas do estudo }\end{array}$ & $\begin{array}{l}5-7 \\
\text { semanas }\end{array}$ & $\begin{array}{l}3 \text { a } 4 x / \text { sem, } 2 x \text { ao } \\
\text { dia. EX aeróbio de } \\
5-10 \text { min com } 40- \\
50 \% \text { da FC } \text { máx } ; 5-15 \text { min de TF no PSE } \\
5 \text { - moderada; } 5-10 \\
\text { min de EX básicos; } \\
\text { caminhada pós alta } \\
\text { hospitalar de } 10-30 \\
\text { min, } \geqslant 3 x / \text { sem }(2 \\
\text { semanas) }\end{array}$ & $\begin{array}{l}\text { Resistência } \\
\text { cardiorrespiratória, } \leftrightarrow \\
\text { resistência muscular } \\
\mathrm{e}^{-} \text {fadiga e depressão. } \\
\text { Tendência de }{ }^{-} \text {IL-6 e IL- } \\
10 \text { com a } \leftrightarrow \text { IFN-y. } \\
02 \text { complicações não } \\
\text { relacionadas ao EX }\end{array}$ \\
\hline
\end{tabular}




\begin{tabular}{|c|c|c|c|c|c|c|c|}
\hline $\begin{array}{l}\text { Citação/ Revista/ } \\
\text { Fator de Impacto }\end{array}$ & $\begin{array}{l}\text { Tipo de } \\
\text { estudo }\end{array}$ & Amostra & Idade & $\begin{array}{c}\text { Intervenção/ } \\
\text { Treinamento físico }\end{array}$ & Duração & $\begin{array}{l}\text { Frequência/ } \\
\text { Intensidade }\end{array}$ & Resultados/Atrito \\
\hline $\begin{array}{l}\text { Klepin et al. } \\
(2010)^{27} / \text { Journal } \\
\text { of Geriatric } \\
\text { Oncology / 1,000 }\end{array}$ & Grupo único & $\begin{array}{l}24 \text { indivíduos } \\
\text { com } \\
\text { LMA - em } \\
\text { tratamento }\end{array}$ & $\begin{array}{l}65,1 \pm 7,8 \\
\text { anos }\end{array}$ & $\begin{array}{l}\text { Grupo único, } \\
\text { EX progressivo } \\
\text { supervisionado: } \\
\text { TC (caminhada + TF) } \\
+ \text { EX flexibilidade }\end{array}$ & $\begin{array}{l}4 \\
\text { semanas }\end{array}$ & $\begin{array}{l}3 x / \text { sem, } 15 \text { min de } \\
\text { caminhada ( até } 15 \\
\text { min) + } 15 \text { min de TF } \\
\text { e EX flexibilidade } \\
+15 \text { min de } \\
\text { caminhada ( até } 15 \\
\text { min) }\end{array}$ & $\begin{array}{l}\text { QV; }{ }^{-} \text {depressão; } \\
\text { desempenho físico. } \\
\text { Nenhum paciente com } \\
\text { complicação relacionada } \\
\text { ao EX }\end{array}$ \\
\hline $\begin{array}{l}\text { Alibhai et } \\
\text { al. }(2012)^{28} \\
\text { / Leukemia } \\
\text { Research / 2,923 }\end{array}$ & Grupo único & $\begin{array}{l}52 \text { indivíduos } \\
\text { com } \\
\text { LMA - em } \\
\text { tratamento }\end{array}$ & $\begin{array}{l}56,4 \pm 12,9 \\
\text { anos }\end{array}$ & $\begin{array}{l}\text { Grupo único, } \\
\text { EX progressivo } \\
\text { supervisionado: } \\
\text { TC (caminhada ou } \\
\text { cicloergômetro + TF) } \\
+ \text { EX flexibilidade }\end{array}$ & 0-17 dias & $\begin{array}{l}4 \text { a } 5 x / \text { sem, } 10 \text { min } \\
\text { de caminhada ( até } \\
40 \text { min) com } 50-75 \% \\
\text { da FCR }+10 \text { min } \\
\text { de TF ( até } 25 \text { min) } \\
\text { com } 50-75 \% \text { da } \\
\text { FCR + } 5-10 \text { min EX } \\
\text { flexibilidade }\end{array}$ & $\begin{array}{l}\text { distância total percorrida } \\
\text { (capacidade funcional } \\
\text { geral); aptidão aeróbica }\left(\mathrm{VO}_{2}\right. \\
\text { pico); - fadiga e ansiedade. } \\
\text { Um possível evento } \\
\text { osteomuscular de grau II } \\
\text { ocorreu (pescoço / costas } \\
\text { função dor limitante para } 24 \\
\text { h). Não há outros eventos } \\
\text { indesejáveis ou preocupações } \\
\text { de segurança relatadas }\end{array}$ \\
\hline $\begin{array}{l}\text { Jarden et } \\
\text { al. }(2013)^{29} \\
\text { / Leukemia } \\
\text { Research / 2,923 }\end{array}$ & Grupo único & $\begin{array}{l}20 \text { indivíduos } \\
\text { com LMA e } \\
\text { LLA - pré-QC }\end{array}$ & $\begin{array}{l}49 \pm 18,2 \\
\text { anos }\end{array}$ & $\begin{array}{l}\text { Grupo único, } \\
\text { EX progressivo } \\
\text { supervisionado: } \\
\text { TC (cicloergômetro } \\
+ \text { TF) }\end{array}$ & $\begin{array}{l}6 \\
\text { semanas }\end{array}$ & $\begin{array}{l}3 x / \text { sem, } 20-25 \text { min } \\
\text { de cicloergômetro } \\
\text { com max. de } 80 \% \text { da } \\
\mathrm{FC}_{\text {máx }}+\mathrm{TF} \text { ( } 2 \text { séries } \\
\text { de } 12 \text { repetições) }\end{array}$ & $\begin{array}{l}\text { distância total percorrida } \\
\text { (capacidade funcional } \\
\text { geral); capacidade física; } \\
\text { QV; - fadiga, náuseas, } \\
\text { angústia, torpor e tristeza. } \\
03 \text { indivíduos não } \\
\text { concluíram o estudo } \\
\text { por falta de motivação/ } \\
\text { agendamento. Nenhum } \\
\text { paciente com complicação } \\
\text { relacionada ao EX }\end{array}$ \\
\hline
\end{tabular}

Legendas: LA, Leucemia aguda; LMA, Leucemia mielóide aguda; LLA, Leucemia linfóide aguda; TCTH, Transplante de células tronco hematopoiéticas; GC, Grupo controle; x/sem, Vezes por semana; GPT3, Grupo de terapia 3; GPT5, Grupo de terapia 5; TC, Treinamento concorrente; TF, Treinamento de força; EX. Treinamento físico; GI, Grupo intervenção; FC, Frequência cardíaca; BPM, Batimentos por minuto; , aumento; ${ }^{-}$, diminuição; $\leftrightarrow$, manutenção; $F C_{\text {máx }}$ Frequência cardíaca máxima; PSE, Percepção subjetiva de esforço; IL, Interleucina; IFN-y, Interferon gama; QV, Qualidade de vida; FCR, frequência cardíaca de reserva; $\mathrm{VO}_{2}$ pico, pico de consumo de oxigênio; QC, Quimioterapia de consolidação; Max., máximo.

Os estudos que aplicaram intervenção apenas com exercício aeróbio ${ }^{26}$, em que ocorreu aumento da distância total percorrida no teste de 12 minutos, redução de angústia e manutenção dos níveis de fadiga. A intervenção que utilizou apenas o treinamento de força ${ }^{25}$ propiciou manutenção dos níveis de proteína muscular.

Os estudos que testaram o treinamento concorrente ${ }^{23,27-29}$ demonstraram melhora ou manutenção dos níveis de fadiga relacionada ao câncer ${ }^{23,28,29}$, além de relatadas melhorias na angustia ${ }^{30}$, ansiedade ${ }^{31}$, qualidade de vida ${ }^{32}$ e depressão $\mathrm{O}^{23,27}$, assim como no desempenho físico ${ }^{23,27-29}$. Battaglini e colaboradores $(2012)^{23}$ ainda avaliaram o impacto da intervenção em marcadores inflamatórios em um grupo único, apontando tendência de redução de interleucina-6 (pró-inflamatória), aumento de interleucina-10 (anti-inflamatória) e manutenção dos níveis de interferon gama com o treinamento físico.

\section{DISCUSSÃO}

Os seis estudos inclusos nessa revisão sistemática, tiveram como objetivo primário verificar a viabilidade de intervenção baseada no exercício físico envolvendo pacientes com leucemia aguda, sendo quatro estudos ${ }^{23,27-29}$ de grupo 
único e dois ${ }^{25,26}$ contando com grupo controle. Os resultados apresentados nestes estudos iniciais são promissores e servem de base para futuras investigações examinando os efeitos de participação regular em exercício físico de pacientes com leucemia aguda durante o tratamento. Apesar do número tão reduzido de estudos realizados até hoje, a viabilidade de implementação de intervenções utilizando exercício físico durante o tratamento é aparentemente possível, mínimos são os efeitos adversos ao exercício físico, e melhoras em algumas capacidades físicas e psicológicas apresentam potencial de serem alcançadas ${ }^{23,25-29}$.

Em relação à viabilidade de implementação de exercício físico em indivíduos com leucemia aguda, todos os seis artigos apresentaram resultados satisfatórios no que diz respeito à tolerabilidade por parte dos pacientes. Apesar de que nem todos os artigos apresentaram medidas objetivas de mensuração de viabilidade da intervenção, poucos efeitos adversos a intervenção foram apresentados. No estudo de Alibhai et al..$^{28}$ um único paciente apresentou dor nas costas, tendo que se afastar do TF pelo período de 24 horas, mas não sendo necessária a sua retirada da intervenção. Em outro estudo, Jarden et al ${ }^{29}$ informaram que três pacientes desistiram da intervenção por falta de motivação e/ou problemas de agendamento, porém não relacionando diretamente ao exercício físico, sendo que nos demais estudos nenhum evento adverso relacionado à pratica do exercício físico foi reportado, corroborando com a ideia de viabilidade da prática do exercício físico por esta população.

Para melhor discutir a viabilidade, devem ser consideradas as modificações positivas físicas e psicológicas que ocorreram na amostra submetida ao exercício físico. Em relação aos efeitos da intervenção nas variações psicológicas, foram observadas diminuição dos sintomas de fadiga, angústia e depressão, bem como melhora da qualidade de vida, quando a intervenção foi baseada em treinamento concorrente (exercício físico aeróbio e de força ${ }^{23,29}$. Quando conciliado o treinamento concorrente aos alongamentos houve redução de sintomas de fadiga ${ }^{23,28,29}$, depressão ${ }^{23,27}$ e ansiedade ${ }^{28}$, bem como aumento da qualidade de vida ${ }^{27}$, porém foram todos estudos de grupo único, e para uma melhor interpretação do real efeito da intervenção por meio do exercício físico em pacientes com leucemia, estudos randomizados com grupo controle são necessários.

Apenas dois estudos com grupo controle ${ }^{25,26}$ foram conduzidos até o momento, porem apenas Chang e colaboradores ${ }^{26}$ avaliaram sintomas de angústia, encontrando reduções desta variável quando comparada ao grupo controle (o qual apresentou aumento desta variável), tendo maior força o achado, vale ressaltar que o estudo teve a duração de apenas 3 semanas e foi baseado somente em exercício físico aeróbio, mas mesmo assim encontrando modificações positivas, possivelmente relacionadas à intervenção.

Quando observada as modificações físicas, os autores que efetuaram o estudo empregando o treinamento físico concorrente ${ }^{23,27-29}$ verificaram que houve aumento na capacidade funcional geral dos pacientes; mais especificamente, melhora da resistência cardiorrespiratória ${ }^{23,28}$, manutenção dos níveis de força $\mathrm{a}^{23}$ e melhora no desempenho físico geral ${ }^{27-29}$.

Cabe ressaltar que as modificações apresentadas não podem ainda serem atribuídas a determinado tipo de intervenção devido à variedade de modalidades, duração e intensidades de treinamentos entre esses estudos, impossibili- 
tando optar por uma ou outra intervenção como possível padrão para treinamento, sendo necessários maiores estudos com amostras mais representativas da população estudada.

Conclui-se que os estudos apontam para a viabilidade e segurança do treinamento físico executado por pacientes com leucemia aguda em tratamento e que possíveis melhoras em parâmetros físicos e psicológicos possam ser alcançados, todavia, mais pesquisas são necessárias buscando uma possível correlação entre os efeitos do treinamento físico sobre a duração da internação, o número de transfusões de sangue, complicações no tratamento, e os efeitos sobre a função imunológica bem como marcadores de inflamação associados a modificações negativas na fisiologia dos pacientes se justificam.

\section{Contribuicão dos autores}

Alan Moraes: Idealizador do artigo participou na busca e seleção de artigos originais nas bases de dados em acordo com metodologia específica da COCHRANE, auxiliou na construção do texto e revisão final; Claudio Battaglini: Providenciou acesso à literatura, auxiliou na construção do texto e revisão final; Ariany Marques Vieira e Douglas Contini Smielewski: Participou na busca e seleção de artigos originais nas bases de dados em acordo com metodologia específica da COCHRANE, auxiliou na construção do texto; Magnus Benetti: Orientou a construção do artigo, participou da revisão final.

\section{Agradecimentos}

Ao Programa do Fundo de Apoio À Manutenção e ao Desenvolvimento da Educação Superior - FUMDES e ao Centro de Estudos Pesquisa e Ensino CEPON.

\section{REFERENCIAS}

1. Ilhan G, Karakus S, Andic N. Risk factors and primary prevention of acute leukemia. Asian Pacific journal of cancer prevention: APJCP. 2006; 7: 515-7.

2. Quintas-Cardama A, Santos FP, Garcia-Manero G. Histone deacetylase inhibitors for the treatment of myelodysplastic syndrome and acute myeloid leukemia. Leukemia. 2011; 25: 226-35.

3. Bolanos-Meade J, Guo C, Gojo I, Karp JE. A phase II study of timed sequential therapy of acute myelogenous leukemia (AML) for patients over the age of 60: two cycle timed sequential therapy with topotecan, ara-C and mitoxantrone in adults with poor-risk AML. Leuk Res. 2004; 28: 571-7.

4. Instituto Nacional de Cancer Jose Alencar Gomes da Silva (Brasil). Estimativa 2012: incidencia de cancer no Brasil. Rio de Janeiro: INCA. 2011; 118p.

5. Dietz K. Acute leukemia. In: Miaskowski C, Buchsel P, eds. Oncology Nursing: Assessment and Clinical Care. St. Louis, MO: Mosby. 1999: 1233-37.

6. Gratwohl A, Baldomero H, Horisberger B, Schmid C, Passweg J, Urbano-Ispizua A. Current trends in hematopoietic stem cell transplantation in Europe. Blood. 2002; 100: 2374-86.

7. Cleeland CS. Cancer-related fatigue: new directions for research. Cancer. 2001; 92(6suppl): 1657-61.

8. Mock V. Evidence-based treatment for cancer-related fatigue. J Natl Cancer Inst Monogr. 2004; 32: 112-8.

9. Jarden M, Hovgaard D, Boesen E, Quist E, Adamsen K. Pilot study of a multimodal intervention: mixed-type exercise and psychoeducation in patients undergoing allogeneic stem cell transplantation. Bone Marrow Transplant. 2007; 40: 793-800. 
10. National Comprehensive Cancer Network Practice Guidelines. Cancer-Related Fatigue Panel 2004 Guidelines, version 1. Rockledge (PA): National Comprehensive Cancer Network. 2004.

11. Pallera AM, Schwartzberg LS. Managing the toxicity of hematopoietic stem cell transplant. J Support Oncol. 2004; 2: 223.

12. Andrykowski MA, Carpenter IS, Greiner CB, Altmaier EM, Burish TG, Antin JH, et al. Energy level and sleep quality following bone marrow transplantation. Bone Marrow Transplant. 1997; 20: 669-679.

13. Molassiotis A, Morris P. Quality of life in patients with chronic myeloid leukemia after unrelated donor bone marrow transplant. Cancer Nurs. 1999; 22: 340-9.

14. Jarden M, Baadsgaard MT, Hovgaard DJ, Boesen E, Adamsen L. A randomized trial on the effect of a multimodal intervention on physical capacity, functional performance and quality of life in adult patients undergoing allogeneic SCT. Bone Marrow Transplant. 2009; 43: 725-37.

15. Courneya KS, Sellar CM, Stevinson C, McNeely ML, Peddle CJ, Friedenreich CM, et al. Randomized controlled trial of the effects of aerobic exercise on physical functioning and quality of life in lymphoma patients. J Clin Oncol. 2009; 27: 4605-12.

16. Carlson LE, Smith D, Russell J, Fibich C, Whittaker T. Individualized exercise program for the treatment of severe fatigue in patients after hematopoietic stem-cell transplant: a pilot study. Bone Marrow Transplant. 2006; 37: 945-54.

17. Wilson RW, Jacobsen PB, Fields KK. Pilotstudy of a homebased aerobic exercise program for sedentary cancer survivors treated with hematopoietic stem cell transplantation. Bone Marrow Transplant. 2005; 35: 721-7.

18. Mello M, Tanaka C, Dulley FL. Effects of an exercise program on muscle performance in patients undergoing allogeneic bone marrow transplantation. Bone Marrow Transplant. 2003; 32: 723-8.

19. Dimeo F, Bertz H, Finke J, Fetscher S, Mertelsmann R, Keul J. An aerobic exercise program for patients with haematological malignancies after bone marrow transplantation. Bone Marrow Transplant. 1996; 18: 1157-60.

20. Baumann FT, Kraut L, Schu“ le K, Bloch W, Fauser AA. A controlled randomized study examining the effects of exercise therapy on patients undergoing haematopoietic stem cell transplantation. Bone Marrow Transplant. 2010; 45: 355-62.

21. Knolls RH, Bruin ED, Uebelhart D, Aufdemkampe U, Schanz F, Stenner-Liewen F, et al. Effects of an outpatient physical exercise program on hematopoietic stem-cell transplantation recipients: a randomized clinical trial. Bone Marrow Transplant. 2010 Dez.

22. Baumann FT, Zopf EM, Nykamp E, Kraut L, Schu“ le K, Elter T, et al. Physical activity for patients undergoing an allogeneic hematopoietic stem cell transplantation: benefits of a moderate exercise intervention. European Journal of Haematology. 2011; 87: 148-56.

23. Battaglini CL, Hackney AC, Garcia R, Groff D, Evans E, Shea T. The effects of an exercise programin leukemia patients. Integr Cancer Ther. Jun 2009; 8(2): 130-8.

24. Higgins JPT, Green S. Cochrane handbook for systematic reviews of interventions. Version 5.1.0 [updated March 2011]. The Cochrane Collaboration. 2011. Disponível em: http://www.cochrane-handbook.org. Acessado em 2012 (10 jun).

25. Cunningham BA, Morris G, Cheney CL, Buergel N, Aker SN, Lenssen P. Effects of resistive exercise on skeletal muscle in marrow transplant recipients receiving total parenteral nutrition. Journal of Parenteral and Enteral Nutrition. 1986; 10(6): 558-63.

26. Chang P, Lai Y, Shun S, Lin L, Chen M, Yang Y, et al. Effects of a walking intervention on fatigue-related experiences of hospitalized acute myelogenous leukemia patients undergoing chemotherapy: a randomized controlled trial. J Pain Symptom Manage. 2008; 5: 524-34.

27. Klepin HD, Danhauer SC, Tooze JA, Stott K, Daleyd K, Vishnevsky T, et al. Exercise for older adult inpatients with acute myelogenous leukemia: a pilot study. Journal of Geriatric Oncology. 2011; 2: 11-7.

28. Alibhai SMH, O’Neill S, Fisher-Schlombs K, Breunis H, Brandwein JM, Timilshina $\mathrm{N}$, et al. A clinical trial of supervised exercise for adult inpatients with acute myeloid leukemia (AML) undergoing induction chemotherapy. Leukemia Research. 2012; 36: $1255-61$. 
29. Jarden M, Adamsen L, Kjeldsen L, Birgens H, Tolver A, Christensen JF, et al. The emerging role of exercise and health counseling in patients with acute leukemia undergoing chemotherapy during outpatient management. Leukemia Research. 2013; 37: $155-61$

30. Coleman EA, Coon S, Hall-Barrow J, Richards K, Gaylor D, Sterwart B. Feasibility of exercise during treatment for multiple myeloma. Cancer Nurs. 2003; 26: 410-9

31. Wiskemann J, Huber G. Physical exercise as adjuvant therapy for patients undergoing hematopoietic stem cell transplantation. Bone Marrow Transplant. 2008; 41: 321-9.

32. Hayes S, Davies PS, Parker T, Bashford J, Newman B. Quality of life changes following peripheral blood stem cell transplantation and participation in a mixed-type, moderateintensity, exercise program. Bone Marrow Transplant. 2004; 33: 553-8.

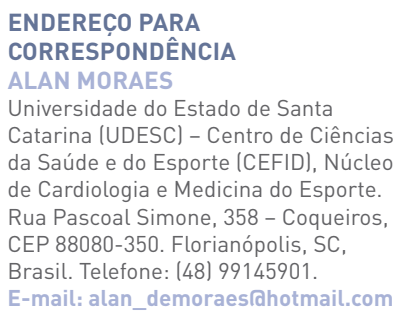

